\title{
La naissance en France de l'enseignement du génie des procédés alimentaires
}

The birth of education in food processes engineering in France

\section{Pierre Vigreux}

\section{OpenEdition \\ Journals}

Édition électronique

URL : http://journals.openedition.org/dht/1776

DOI : $10.4000 /$ dht. 1776

ISSN : $1775-4194$

Éditeur :

Centre d'histoire des techniques et de l'environnement du Cnam (CDHTE-Cnam), Société des élèves du CDHTE-Cnam

\section{Édition imprimée}

Date de publication : 1 décembre 2011

Pagination : 135-145

ISBN : 978-2-9530779-7-1

ISSN : 0417-8726

Référence électronique

Pierre Vigreux, «La naissance en France de l'enseignement du génie des procédés alimentaires », Documents pour I'histoire des techniques [En ligne], 20 | 2e semestre 2011, mis en ligne le 17 septembre 2012, consulté le 08 septembre 2020. URL : http://journals.openedition.org/dht/1776 ; DOl : https:// doi.org/10.4000/dht.1776 


\title{
La naissance en France de l'enseignement du génie des procédés alimentaires
}

Pierre Vigreux

\begin{abstract}
Résumé
La notion d'« opérations unitaires» a permis, dans les années soixante, une approche scientifique des techniques utilisées dans les industries agro-alimentaires. Cette discipline est issue du génie chimique dont les origines se situent à la fois en Europe et aux États-Unis, tout particulièrement au MIT. En France, cet enseignement, a été dispensé d'abord à l'École Nationale Supérieure des Industries agricoles et alimentaires (ENSIA), créée à la fin du XIXe siècle, pour répondre auX besoins de l'industrie sucrière. Cet enseignement qui doit beaucoup à Marcel Loncin, ingénieur d'origine belge. est devenu dans les années quatre-vingt le « génie des procédés alimentaires ». En 2007, l'ENSIA s'est intégré à l'Institut des sciences et industries du vivant et de l'environnement, dénommé Agro Paris Tech. Ceci a donné à cet enseignement un cadre institutionnel plus large.
\end{abstract}

Résumés et mots clés en anglais sont regroupés en fin de volume, accompagnés des mots clés français

L 'enseignement, en France, des sciences et des

Lechniques nécessaires à ce qu'il est convenu d'appeler, à l'heure actuelle, les industries agroalimentaires, qui sont plus généralement les industries de transformation des produits agricoles en produits alimentaires, s'est fait par étapes. La plus importante nous paraît être la mise en place de l'enseignement du génie des procédés alimentaires. Plus particulièrement il s'agit de montrer comment cet instrument de description des techniques que constitue le concept d' "opération unitaire " a permis de mettre de la cohérence dans l'enseignement des sciences et des techniques mises en œuvre dans ces industries que, pour la clarté de l'exposé, nous désignerons dans la suite de cet article sous le terme de sciences pour l'industrie alimentaire.

Dans ce but, nous préciserons ce qu'est la notion d'opérations unitaires qui donné naissance au génie chimique puis tout récemment au génie des procédés. Nous rappellerons également les conditions de la création de l'institution dans laquelle s'est mise en place un enseignement des sciences pour l'industrie alimentaire. II s'agit de l'École nationale supérieure des industries agricoles et alimentaires (ENSIA), aujourd'hui fusionnée dans un en ensemble plus vaste qui sera présenté très brièvement à la fin. Ensuite, sera présentée la rupture qu'a constituée la Deuxième guerre mondiale, et enfin les conditions dans lesquelles s'est mise en place, en France, cet enseignement du génie des procédés alimentaires.

\section{La notion d'opération unitaire}

Il ne s'agit aucunement de faire ici un historique du génie chimique qui a été fait par ailleurs mais de présenter la notion d'opération unitaire'. C'est dans la seconde moitié du XIX $X^{e}$ siècle et au début du $X X^{e}$ siècle que plusieurs chimistes ont mis en évidence que, dans les opérations de l'industrie chimique, on retrouvait des opérations similaires.

La prise de conscience de la notion d'opération unitaire II nous paraît, tout d'abord, nécessaire de rappeler, les auteurs qui ont les premiers mis en évidence les similitudes des opérations rencontrées dans les techniques employées dans l'industrie chimique.

\footnotetext{
1 Pour cette première section nous nous sommes tout particulièrement référés à l'ouvrage suivant : Jacques Breysse, Du génie chimique au génie des procédés : émergence d'une science pour l'ingénieur (1947-1991), Mémoire de DEA, Cnam-Centre d'histoire des techniques, septembre 2004.
} 
Nous retiendrons un de ces auteurs dans chacun des 3 grands pays qui comptaient à l'époque en ce domaine. Le cas des Etats-Unis étant différent, nous citerons, ensuite, ceux qui ont participé à cette prise de conscience. En Allemagne, il s'agit de Alwyn Parnicke2, au Royaume-Uni de Georges E. Davis (1850-1907) ${ }^{3}$ et en France d'Eugène Péclet (17931857)4. Aux Éłats-Unis, alors qu'Arthur D. Little (18631935) a le mérite dans un rapport de 1915 d'employer pour la première fois le terme d'u unit operations ॥ (opérations unitaires), il faut attendre 1923 pour que trois professeurs du Massachusetts Institute of Technology (MIT), Wiliam H. Walker (1869-1908), Warren K. Lewis (1882-1975) et Wiliam H. Mac Adams publient un ouvrage qui est considéré comme le traité fondateur de cette démarche 5 . Cet ouvrage systématise la notion d'« opérations unitaires ॥ dans l'approche scientifique de l'industrie chimique. Les têtes de chapitre de cet ouvrage sont rappelées dans le tableau I (colonne 1). Ce tableau met en parallèle dans ses trois dernières colonnes, les têtes de chapitre des traités des auteurs européens cités plus haut. On relève le parallélisme de ces différents traités. Par exemple des opérations très simples telles que le broyage sont cités à la ligne 7, l'évaporation à la ligne 10 et le séchage à la ligne 12. À la ligne 13 c'est une opération plus élaborée, la distillation qui est étudiée dans les quatre traités. Cette discipline qui reçoit aux États-Unis la dénomination de " chemical engineering " consiste à étudier de manière approfondie chacune de ces « opérations unitaires " et à bénéficier de l'expérience acquise dans différentes industries pour une même opération. Ceci n'épuise cependant pas l'approche scientifique de l'industrie chimique.

Le développement de cette approche scientifique aux États-Unis

\| convient de rappeler qu'aux États-Unis au MIT, durant l'année scolaire 1907-1908, William H. Walker

2 Alwyn Parnicke, Des aides mécaniques pour l'industrie chimique (Die maschinellen hilfsmittelder chemischen technik), Francfort, Bechold, $1^{\mathrm{e}}$ édition 1894, $2^{\mathrm{e}}$ édition 1898, traduction française 1906.

3 Georges E. Davis, Manuel de génie chimique (Handbook of chemical engineering), $1^{e}$ édition 1901, $2^{e}$ édition 1898 , New York/Paris, Mc Graw-Hill/Dunod.

4 Eugène Péclet, Traité de la chaleur considérée dans ses applications, Hachette, Paris, $2^{e}$ édition 1843.

5 Wiliam H. Walker, Warren K. Lewis, Wiliam H. MacAdams, Principes de chimie industrielle (Principles of chemical engineering), New-York, 1923. Traduction française d'après la $2^{\mathrm{e}}$ édition, Paris, Dunod, 1933. déjà cité, inaugure un cours qui reprend cette approche par opérations unitaires. Simultanément se crée l'American Institution of Chemical Engineers (AIChE). Cette date représente, donc, dans ce pays, I'institutionnalisation de cette discipline.Ultérieurement au cours de la Première Guerre mondiale, les ÉtatsUnis notamment du fait de leur entrée tardive dans la guerre, se sont trouvés devant la nécessité d'adapter leur industrie chimique aux conditions de la guerre, non seulement pour satisfaire leurs propres besoins, mais également pour approvisionner leurs alliés, alors que leur industrie était jusque là dépendante de l'Allemagne. C'est pourquoi, afin d'optimiser leur industrie chimique ils ont dû faire appel aux méthodes du " chemical engineering " qui ont, de ce fait, reçu la sanction d'une pratique intensive. Ce sont, d'une part, le rôle scientifique jové par le MIT, notamment par le traité fondateur de 1923 et, d'autre part, l'intensification de l'industrie chimique nécessitée par leur entrée tardive dans la guerre qui expliquent que l'approfondissement de cette discipline scientifique se poursuive aux États-Unis et non dans l'un des trois pays européens cités plus haut, malgré leur avance sur le plan conceptuel.

Sur le plan sémantique, les Canadiens vont traduire le terme de "chemical engineering " par "génie chimique " ce qui va devenir l'appellation usuelle de cette discipline en langue française jusqu'aux années quatre vingt. Nous remarquons que, de ce fait, l'ouvrage de 1923 est traduit en Français de manière impropre puisqu'il aurait dû être dénommé : Traité de génie chimique et non Traité de chimie industrielle.

Le cas de Léon Lindet

Pour être complet, il convient de rappeler ici le cas de Léon Lindet.(1857-1929), professeur de technologie agricole à l'Institut national agronomique. L'intéressé publie en 1922 un ouvrage dans lequel il adopte une démarche similaire. ${ }^{6}$ En partant de l'étude des appareils, Lindet identifie ce qu'il appelle des " groupes d'opération » tels que la concentration, la dessication, la distillation. Léon Lindet est conscient de l'aspect novateur de sa démarche puisqu'il précise dans l'avant-propos : « je pense que c'est la première classification méthodique que l'on publie des appareils de l'industrie chimique $»$. Cet ouvrage n'aura malheureusement aucun retentissement, mis à part un article dans le Bulletin des chimistes, publication de l'Association des chimistes de sucrerie

6 Léon Lindet, L'outillage de l'industrie chimique, agricole et alimentaires, Paris, Eyrolles, 1922. 
Comparaison des traités fondateurs du génie chimique

Tableau 1

E. Péclet G. Davis

\begin{tabular}{|c|c|c|c|}
\hline $\begin{array}{c}\text { Walker,Lewis } \\
\text { and Mac Adams } \\
1923\end{array}$ & $\begin{array}{c}\text { E. Péclet } \\
\text { ( } 2 \text { e éd. } 1843 \text { ) }\end{array}$ & $\begin{array}{c}\text { G. Davis } \\
\text { (2é éd. 1901) }\end{array}$ & $\begin{array}{c}\text { A. Parnicke } \\
1906 \\
\text { base 2é éd. } 1898 \\
\end{array}$ \\
\hline $\begin{array}{l}\text { Elements of industrial } \\
\text { stoichiometry, proprietes } \\
\text { physiques, bilans. }\end{array}$ & - & & (2) \\
\hline $\begin{array}{l}\text { Fluiid films, Flow offluids } \\
\text { mesures des vitesses, } \\
\text { viscøsité, diagrammes des } \\
\text { pertes de charges. }\end{array}$ & Ecoulement des gaz comprimés & $\begin{array}{c}\text { Moving liquids, solids and gases } \\
\text { absorbing and compressing } \\
\text { gases. }\end{array}$ & $\begin{array}{l}\text { Trasport des solides, } \\
\text { des liquides et des gaz }\end{array}$ \\
\hline $\begin{array}{c}\text { IFlow of heat } \\
\text { comduction, convection, } \\
\text { radiation }\end{array}$ & $\begin{array}{l}\text { Théorie physique de la chaleur. } \\
\text { Chauffage des solides. } \\
\text { Refroidissement. Mouvement de } \\
\text { l'air chaud. Cheminées. }\end{array}$ & $\begin{array}{l}\text { The application of heat } \\
\text { and cold }\end{array}$ & \\
\hline $\begin{array}{l}\text { Fuels and power, } \\
\text {.combustion. }\end{array}$ & $\begin{array}{l}\text { Combustion } \\
\text { et combustibles. }\end{array}$ & $\begin{array}{l}\text { Steam production, power } \\
\text { and its application. }\end{array}$ & $\begin{array}{l}\text { Production de force motrice } \\
\text { Machines à vapeur,moteurs } \\
\text {.électriques. } \\
\end{array}$ \\
\hline Fiurnace and kilns & $\begin{array}{c}\text { Foyers } \\
\text { Chauffage des liquides. }\end{array}$ & & $\begin{array}{l}\text { Fours, autoclaves } \\
\text { chap.: Fusion, dissolution, } \\
\text { lixiviation. }\end{array}$ \\
\hline \multicolumn{4}{|l|}{$\begin{array}{l}\text { Gas producers } \\
\text { "gaz a leau" }\end{array}$} \\
\hline Griinding and Crushing. & & Treating and preparing solids. & Appareils de broyages. \\
\hline $\begin{array}{l}\text { Mechanical separation } \\
\text { "décantation, gravité " } \\
\text { filtration. }\end{array}$ & & $\begin{array}{l}\text { Separation solubles from } \\
\text { insolubles. }\end{array}$ & $\begin{array}{c}\text { Filtration / décantation } \\
\text { chap.: procédés mécaniques } \\
\text { de séparation des corps }\end{array}$ \\
\hline $\begin{array}{c}\text { Basic principals } \\
\text { of vaporization process. }\end{array}$ & Vaporization. & & \\
\hline Evaporation. & Evaporation. & $\begin{array}{l}\text { Evaporation } \\
\text { and distillation. }\end{array}$ & $\begin{array}{c}\text { Evaporation } \\
\text { Chap.: concentration. }\end{array}$ \\
\hline \multicolumn{4}{|l|}{$\begin{array}{l}\text { Humidity and wet and bulb } \\
\text { thermometry, humidifiers, } \\
\text { deshumudifiers and water cooler }\end{array}$} \\
\hline Drying. & Séchage. & & Dessication. \\
\hline \multirow[t]{9}{*}{ Distillation. } & Distillation. & Evaporation and distilation. & $\begin{array}{l}\text { Distillation chap.: procédés } \\
\text { méca. séparation des corps. }\end{array}$ \\
\hline & Chauffage de l'air. & & \\
\hline & $\begin{array}{l}\text { Chauffage, ventilation } \\
\text { et assainissement. }\end{array}$ & & $\begin{array}{c}\text { Ventilation et élimination } \\
\text { des pousières. }\end{array}$ \\
\hline & & Matérials in plant construction & \\
\hline & & Weighing and measuring. & Appareils de contrôle. \\
\hline & & Cristallisation and dialysis. & $\begin{array}{l}\text { Cristallisation chap. : procédés } \\
\text { méca séparation des corps. }\end{array}$ \\
\hline & & $\begin{array}{l}\text { Electrolysis and electro- } \\
\text { smelting. }\end{array}$ & \\
\hline & & The constuction of packages. & \\
\hline & & & Mélangeurs. \\
\hline
\end{tabular}

Extrait de : Jacques Breysse, Du génie chimique au génie des procédés (1947 - 1991), 2004

et de distillerie qui sera présentée plus loin, ce qui est assez compréhensible quand on sait que Lindet était membre de cette association depuis 1894 et qu'il avait assumé la présidence de 1895 à 1897 ainsi que de 1919 à 1921.

Deux explications peuvent être proposées à cet oubli dans lequel est tombé cet ouvrage. La première tient au fait que les milieux scientifiques paraissent davantage préparés à développer une approche partant de la composition chimique des corps plutôt que des matériels utilisés ${ }^{7}$. La seconde résulte

7 Léon Lindet étant membre de l'Académie des sciences avait déposé un exemplaire de cet ouvrage à la biblio- de l'attitude des milieux économiques concernés en l'occurrence les organisations professionnelles de filières telles que la sucrerie ou la brasserie qui n'entretiennent, le plus souvent, que très peu de relations entre elles ce qui n'est pas pour favoriser une approche transversale, c'est à dire commune à plusieurs filières ${ }^{8}$. II est très regrettable que les concepts mis en avant âr Loncin soient tombés dans

thèque de l'Institut. Or en 1990 quand nous l'avons consulté nous avons eu le triste privilège d'en couper les pages!

8 Le cas de la sucrerie et de la distillerie constitue à cette époque une exception pour des raisons qui seront présentées plus loin. 
l'indifférence car cela aurait permis de donner à l'industrie agro-alimentaire française une avance certaine.

Cette notion d'opération unitaire n'aurait toutefois pas pu se développer en France sans une institution qu'il nous faut maintenant présenter.

\section{La sucrerie de betteraves, l'ENSIA et l'émergence d'une} science pour l'industrie alimentaire

La spécificité de la sucrerie de betteraves

L'École national supérieure des industries agricoles et alimentaires (ENSIA) a été créée, à l'origine, pour faire face aux besoins de l'industrie du sucre de betteraves. Pour comprendre le contexte tout à fait particulier dans lequel se trouve cette industrie vers 1880, il convient de rappeler que cette technique présente une double caractéristique. En effet, d'une part la science y a précédé la technique puisque c'est en 1747 que le chimiste Marggraff propose le premier une méthode pour en extraire le sucre de la betterave, alors que ce n'est qu'en 1786 que son disciple Achard entreprend d'industrialiser cette fabrication. D'autre part, l'industrie y a précédé l'agriculture puisque à la suite du Blocus continental, des sucreries se sont installées avant qu'on ne cultive des betteraves sucrières aux alentours. II y avait donc, dans cette industrie de transformation de produits agricoles en produits alimentaires, à la différence des autres, à la fois, la conscience du fait que cette industrie était directement issue de la découverte d'un chimiste et, également, le dynamisme de l'industrie motrice dans cette activité alors que les autres industries similaires de transformation restaient de nature artisanale ou agricole. Ce contexte favorise un approfondissement scientifique et technique, animé essentiellement en France par les chimistes ${ }^{9}$

En effet, à cette époque, la chimie prend de plus en plus d'importance dans la sucrerie et la distillerie, à la fois à des raisons fiscales et techniques. D'une part, l'impôt étant perçu par catégorie de richesse, il est de l'intérêt de l'industriel de s'approcher le plus possible de la limite, tout en évitant d'atteindre la catégorie supérieure pour laquelle l'impôt est le plus élevé ${ }^{10}$. D'autre part plusieurs innovations techniques

9 Pour tout ce qui concerne I'ENSIA dans cet article, voir : Pierre Vigreux, La naissance, le développement et le rôle de l'École nationale supérieure des industries agricoles et alimentaires (ENSIA) 1893-1986, thèse de doctorat en histoire dirigée par Albert Broder et soutenue à I'Université Paris XII le 9 mars 2001

10 La question nous paraît avoir été très bien présentée dans un historique établi en 1927 : « Le problème était celui-ci : étant donné que pour une richesse de 0 à 6\%, l'impôt était le introduites alors nécessitent d'être suivies par des chimistes : il s'agit notamment de la diffusion, de la double carbonatation et de l'évaporation à triple effet ${ }^{11}$ - L'évaporation à triple effet a pur but de concentrer le jus sucré épuré. Cependant ces chimistes se trouvent dans une situation précaire car, en général, recrutés uniquement pour une saison de trois mois. C'est pourquoi, à l'occasion d'un congrès sucrier tenu en 1882, plusieurs chimistes, décident de fonder l'Association des chimistes de sucrerie et de distilleries de France et des colonies, laquelle existe encore aujourd'hui sous le nom d'Association des chimistes, ingénieurs et cadres des Industries agricoles et alimentaires (ACIA). Cette prise en compte de la distillerie par cette association s'explique par l'utilisation d'un même produit agricole d'origine : la betterave sucrière. Ainsi se crée un lieu de réflexion concernant deux filières de transformation. Cette association demande, dès 1886, l'amélioration de la formation des chimistes afin de mettre fin à la précarité de leur situation. Cette action est relayée en Belgique par une association similaire. On ne saurait trop souligner la modernité de cette approche qui consiste à remédier à la précarité d'une situation professionnelle par un complément de formation.

La mise en place de l'ENIA (1882-1939)

Cette école, créée sous l'appellation d'École nationale des industries agricoles (ENIA), n'est cependant pas la première, en Europe, à enseigner ce type de techniques puisqu'elle a été précédée, en particulier en Allemagne en 1876, par l'École de sucrerie de Brunswick et, en France en 1888, par l'École nationale d'industrie laitière de Mamirolle (Doubs). Néanmoins ce titre présente pour l'époque une nouveauté considérable car il manifeste, dès 1893, I'intention de ses promoteurs d'instaurer un enseignement rendant compte de l'ensemble des techniques de transformation des produits agricoles en produits alimentaires et plus particulièrement ce qu'on appelle à l'heure actuelle les industries de première transformation. D'autre part, il convient de

même aux 100 kilos de sucre, les fabricants devaient :

$1^{\circ}$ nécessairement ne pas dépasser la limite supérieure 6 sous peine de payer un impôt plus élevé ; $2^{\circ}$ s'approcher le plus possible de 6 puisque l'impôt était réparti pour 100 kilos de sucre brut sur une plus grande quantité de sucre pur $»$.

11 La diffusion est l'opération qui consiste à extraire le jus sucré à partir des betteraves réduites en fines lames : les cossettes. La double carbonatation a pour but d'épurer le jus sucré, précédemment chaulé, en y faisant barboter du gaz carbonique afin de précipiter l'excès de chaux en carbonate de calcium lequel entraîne les impuretés. 
signaler qu' un professeur à l'Institut agronomique, Aimé Girard (1830-1898), émet dès 1887 l'idée d'un enseignement de technologie agricole comparée, ainsi que l'on désignait à l'époque ces techniques, enseignement obtenu en installant « les uns à côté des autres " des laboratoires à échelle industrielle, relatifs à plusieurs filières de transformation. C'est la convergence de ces préoccupations et de celle des chimistes exposée plus haut qui va fonder le projet pédagogique de l'ENIA.

C'est un événement tout à fait extérieur aux préoccupations industrielles, en l'occurrence la nécessité de dédommager la ville de Douai privée de ses Facultés de droit et de lettres transférées à Lille en 1887, qui a décidé de l'implantation de cette école dans le Nord, Cette implantation a conduit à privilégier, à l'origine, l'enseignement, non seulement de la sucrerie et de la distillerie, mais également de la brasserie-malterie qui était à l'époque une industrie très développée dans le Nord de la France. Mais I'ENIA va connaître très rapidement des difficultés du fait, d'une part, de son implantation dans le centre de la ville et, d'autre part, d'une diminution de dynamisme de l'industrie sucrière. À la suite d'une convention signée à Bruxelles en 1902 entre les pays producteurs de sucre, une cartellisation de cette industrie s'instaure, rendant la concurrence moindre. II est à noter, par ailleurs, que dès les premières années le directeur relève que la juxtaposition d'enseignements par filières conduit à des doubles emplois. Cependant le rapprochement de la distillerie et de la brasserie va se révéler scientifiquement fécond car ces deux industries sont le siège d'opérations de fermentations qui seront approfondies également par l'Institut Pasteur tout particulièrement dans une publication, les Annales de la brasserie et de la distillerie.

La Première Guerre mondiale, en faisant prendre conscience de la dépendance de la France en matière d'approvisionnement pétrolier vis-à-vis de l'extérieur, va se révéler bénéfique pour l'ENIA qui est, à l'époque, la seule école où est enseignée la distillerie industrielle. Cette discipline devient l'enseignement moteur car la communauté ENIA prend conscience que cette dépendance de la France en carburant peut contraindre le Pays à avoir recours à un carburant d'origine nationale. Dans cette hypothèse, I'ENIA pourrait avoir un rôle essentiel à jover. C'est ce qui explique l'arrivée à la direction de l'École, en 1934, d'un jeune directeur Étienne Dauthy, ingénieur agronome qui a commencé sa carrière dans une distillerie. Ce nouveau directeur va incontestablement relever le dynamisme de l'école.
La double origine d'une science pour l'industrie alimentaire

Cette science pour l'industrie alimentaire en cours d'élaboration_présente ainsi une double origine. Il existe, d'abord, une origine franco-belge : la réflexion scientifique et technique se poursuit entre l'Association des chimistes française et celle de Belgique, et donne lieu à un Congrès international technique et chimique de sucrerie et de distillerie, tenu à Liège en 1905, puis à Paris en 1908. Interrompus par la première Guerre mondiale, ces congrès reprennent en 1934 à Paris, sous l'appellation de Congrès des industries agricoles, manifestant ainsi une volonté d'extension à l'ensemble de ces industries. On relève que, dans cette approche européenne, la transformation des produits agricoles, c'est-à-dire l'amont, a été historiquement privilégié par rapport à l'aval puisqu'elle a sa source dans la sucrerie de betterave, industrie de première transformation.

A la même période aux États-Unis et de manière tout à fait indépendante, des travaux scientifiques préparent la naissance d'une approche scientifique des industries alimentaires. C'est au sein du département de biologie du Massachusetts Institute of Technology (MIT) que Samuel C. Prescott commence, en 1904, une série de cours consacrés aux produits alimentaires sous le terme de "industrial biology " et qu'il ouvre, dès 1905, un laboratoire commercial consacré aux questions d'alimentation. La Première Guerre mondiale met en évidence l'importance de l'approvisionnement des armées ainsi que celui des populations. Les divers organismes responsables de cette question et, parmi eux la Food Administration que dirige, à partir de 1917, Herbert Hoover, futur président des Etats-Unis de 1928 à 1932, manifestent en cette circonstance leur intérêt pour cette discipline et contribuent financièrement aux recherches du MIT. Après la Première Guerre mondiale, le MIT adopte l'expression de " food technology ».pour désigner les applications du génie chimique à l'industrie alimentaire. Par ailleurs, le ministère américain de l'Agriculture et plusieurs Universités (Californie, Illinois, Wisconsin, lowa) s'intéressent à cette discipline. À l'initiative des enseignants de MIT, deux congrès internationaux ont lieu à Cambridge (Massachusetts), l'un en 1937 et l'autre en juin 1939. À l'issue de ces travaux est créé l'Institute of Food Technologists dont le siège s'établit à Chicago. II se crée ainsi une "école américaine ", animée plus particulièrement par le MIT. Ce centre de réflexion dont la naissance est de vingt ans postérieure à celle de l'Association des chimistes, privilégie historiquement l'aval, c'està-dire les produits alimentaires. 
La rupture de la Deuxième Guerre mondiale (1940-1960) Dans cette section sont présentées, non seulement les événements ayant influencé cette question intervenus au cours de la Deuxième Guerre mondiale proprement dite, mais également ceux intervenus dans les années de l'après guerre. Le conflit, rendant cruciale la question du ravitaillement, souligne l'importance des questions d'alimentation. Ces circonstances s'avèrent décisives à la fois pour I'ENIA et pour l'enseignement des sciences pour I'industrie alimentaire. Mais parallèlement les milieux scientifiques s'ouvrent, en France, à la démarche du génie chimique.

Conséquences en France sur l'enseignement du Génie chimique

C'est le professeur Joseph Cathala (1892-1969) qui le premier introduit cet enseignement en France. Après avoir été, à partir de 1914, assistant au Collège de France de 1927 à 1931, il exerce au Canada où il prend connaissance du " chemical engineering " et de sa traduction canadienne. Rentré à Toulouse en 1931 il tente, sans succès, d'introduire l'enseignement de cette discipline. En 1940, il exerce son activité en Angleterre comme ingénieur conseil de la Royal Ordonance Factory (ROF) et plus particulièrement dans les poudrières. Revenu en France en 1947, il obtient très rapidement, la même année la création du diplôme d'ingénieur du génie chimique. Puis il propose la mise en place d'un Institut du génie chimique (IGC) qui est finalement créé en 1949 à Toulouse. Presque simultanément le Professeur Maurice Letort (1907-1972), nommé en 1946 directeur de l'École nationale des industries chimiques (ENSIC) de Nancy, y instaure en 1949 un enseignement de génie chimique.

On relève que Joseph Cathala avait vainement essayé, en 1931, d'introduire ce type d'enseignement et que, par ailleurs, il a pendant la guerre exercer son activité dans une poudrière, activité directement liée au conflit.

Conséquences sur la science pour l'industrie alimentaire

C'est tout d'abord aux États-Unis que les recherches se poursuivent notamment au MIT. D'autre part I'Institute of Food Technologists, déjà cité, publie à Chicago à partir de 1947 la revue Food Technology. Enfin en 1950, G. G.Brown publie l'ouvrage Unit opérations dans lequel il approfondit cette question.

La Deuxième guerre mondiale est également la raison pour laquelle un jeune ingénieur chimiste s'oriente vers l'industrie alimentaire et y joue un rôle fondamental. D'origine belge, Marcel Loncin (1920-1994), fait d'abord des études d'ingénieur chimiste qu'il termine en 1940. La Belgique venant alors d'être envahie, Loncin décide de prolonger ses études, s'orientant vers les industries alimentaires, ces questions lui paraissant devoir prendre une importance vitale. Loncin entreprend une double carrière, d'une part d'enseignant à l'Institut des fermentations de Bruxelles et, d'autre part, de directeur technique, d'abord d'une brasserie puis d'une laiterie. II ne cessera de poursuivre cette double activité. Loncin entreprend ensuite des travaux sur les corps gras qui se traduisent par une thèse soutenue à Paris en 1953 et portant sur " L'étude de l'altération de l'huile de palme $"$.

De par sa formation de chimiste, d'abord, par ses fonctions d'enseignant, ensuite, Loncin connaît le concept d'opérations unitaires dans l'approche nordaméricaine du génie chimique. II se rend compte que dans les diverses filières de transformation de produits agricoles en produits alimentaires telles que la laiterie, la brasserie ou la sucrerie, il est possible d'individualiser des opérations qui se retrouvent dans plusieurs de ces filières telles que la filtration, la diffusion ou l'évaporation. II entreprend alors d'appliquer à ces différentes opérations ce concept d'opérations unitaires ainsi que les outils mathématiques mis au point aux États-Unis par le génie chimique. Parallèlement son expérience d'opérateur industriel, lui permet de se rendre compte que cette démarche se révèle opératoire, donc techniquement féconde. L'originalité de Marcel Loncin est de comprendre que cette nouvelle discipline doit disposer d'un traité fondamental qu'il publie en 1961. Les têtes de chapitres, du moins du 2 au 10 telles qu'elles ont présentées dans le tableau II, font clairement ressortir l'approche retenue. Le tableau II présente, en regard des têtes de chapitre de l'ouvrage de Loncin, celle de l'ouvrage de Léon Lindet, paru en 1922 et présenté ci-dessus. La similitude d'approche est flagrante et montre que Lindet avait été véritablement un précurseur. II est certain, par ailleurs, que Loncin n'avait pas eu connaissance de cet ouvrage.

En Europe, plus généralement, la réflexion sur la science pour l'industrie alimentaire se poursuit dans les congrès internationaux des industries agricoles qui reprennent et se tiennent successivement en 1948 à Paris, en 1950 à Bruxelles et en 1952 à Rome. Ce dernier congrès est marqué en particulier par une intervention de Robert Bousser, professeur d'industries agricoles à l'École d'agriculture de Meknès et qui a été, pendant la guerre, officier chargé du ravitaillement au 


\section{Correspondance entre les têtes de chapitres \\ de l'ouvrage de $\mathbf{L}$. Lindet et de celui de M. Loncin}

\section{L'outillage de l'industrie chimique \\ (L. Lindet - 1922)}

VI - La mise en contact des corps solides et gazeux en vue de mélanges, dissolutions et de réactions.

III - La séparation mécanique des éléments entre eux.

IV - Le chauffage.

VIII - La concentration.

$X$ - La distillation.

XI - La sublimation ou distillation sèche

IX - La dessiccation

II - La division de la matière

Non traité

I - Le transport et la manutention.

$\mathrm{V}$ - La réfrigération.

VII - La dissociation et la cuisson à haute température.

XII - La pyrogénation

XIII - La condensation

XIV - La mise en forme et le façonnage

$\mathrm{XV}$ - Le contrôle et la régulation des opérations
Les opérations unitaires du génie chimique

(M. Loncin - 1961)

1 - Généralités sur les transferts

3, 4, 5 - Décantation, filtration.

6 - Pasteurisation et stérilisation.

7 - Évaporation.

8 - Distillation.

Ibid.

9 - Séchage

10 - Broyage, classement, mélange.

11 - Matériaux

\section{Non traité \\ Non traité}

Renseignements manquants

Renseignements manquants

Renseignements manquants

Renseignements manquants

Non traité
Maroc, fonction dans laquelle il a pu découvrir l'importance des moyens mis en œuvre pour l'approvisionnement des armées. Robert Bousser expose que c'est toujours une matière première d'origine biologique qui est transformée et qu'à cette unité doit correspondre une unité des méthodes employées pour cette transformation. À partir du congrès de 1954 tenu à Madrid ces manifestations s'étendent explicitement aux industries alimentaires. Robert Bousser y reprend 
les analyses déjà exposées en 1952 et le directeur de l'ENIA, André Bonastre, y présente un rapport sur l'enseignement dans son école de ce qu'il appelle : «le génie industriel $»^{12}$.

\section{Les conséquences pour l'ENIA}

Les conséquences directes de la Deuxième Guerre mondiale pour l'École sont favorables pour deux raisons. La première est liée à la localisation de l'École dont le fonctionnement est devenu impossible à Douai. Le directeur utilise cette circonstance pour l'implanter à Paris, ce qui la rend plus attractive et relève son niveau. La seconde est que I'ENIA saisit cette opportunité et élargit son enseignement à presque toutes les filières de transformation de produits agricoles en produits alimentaires. Du fait de cette extension, la durée des études est portée de deux à trois ans.

Ce contexte provoque, en outre, un débat sur la formation des cadres des industries agricoles et alimentaires. Ce sont d'abord les professionnels de l'alimentation qui prennent conscience, dès la Libération, de l'importance de leur activité et se préoccupent de la formation du personnel et des cadres en particulier. II faut ajouter que l'Institut national de la recherche agronomique (INRA) créé en 1946, choisit de ne s'intéresser qu'aux filières n'ayant pas encore de caractère industriel. Ceci laisse le champ libre à l'ENIA dans le domaine des industries alimentaires. Plus généralement, ce qui ressort de cette période est l'affirmation de l'unité des industries agricoles et alimentaires.

La prise de conscience de cette unité va, ensuite, se répercuter sur le plan pédagogique à I'ENIA. Cette démarche apparaît en 1951 sous le terme de " technologie comparée ». "I ne s'agit plus de partir d'une filière, elle-même centrée sur un produit et d'analyser les principaux procédés mis en œuvre, mais au contraire, à partir de ces procédés, d'étudier comment ils interviennent dans les différentes filières. Remarquons que, si l'expression de technologie comparée n'apparaît qu'en 1951, la démarche est, elle-même « consubstantielle » de l'École, puisqu'elle était implicitement celle-là même qu'Aimé Girard adoptait en 1887. L'usage de cette expression est éphémère, mais la démarche intellectuelle se

12 André Bonastre, "le génie industriel appliqué aux industries agricoles et alimentaires à l'École nationale des industries agricoles et alimentaires ॥ et Robert Bousser, "Importance, unité et développement des industries agricoles et alimentaires ou industries de conservation et transformation $॥$, dans Commission internationale des industries agricoles et alimentaires, Compte rendu du Xe Congrès, Madrid, 1954. prolonge dans le « Génie industriel », expression qui apparaît dès 1952 à l'École. Au congrès de Madrid en 1954, le directeur de l'ENIA, André Bonastre, définit cette discipline nouvelle. II rappelle d'abord comment, face aux risques de redites qu'entraînait la juxtaposition de l'enseignement de nombreuses filières des industries agricoles et alimentaires, un savoir unificateur s'est avéré nécessaire. Se référant au génie chimique, pour lequel un outil mathématique est déjà au point, I'ENIA définit des « opérations élémentaires ॥. La prise de conscience de l'utilité de l'approche à partir de ce dernier concept émerge donc au sein de l'école parallèlement à celle de Marcel Loncin présenté ci-dessus. Cette extension de fait, de l'École aux industries alimentaires dont nous avons vu qu'elle s'est également traduite dans la nouvelle appellation des congrès internationaux des industries agricoles et alimentaires va, enfin, avoir des conséquences réglementaires et administratives. En effet, l'École va, d'abord en 1954, recevoir l'appellation d'École nationale des industries agricoles et alimentaires. Ultérieurement la loi du 2 août 1960 qui refond l'ensemble de l'enseignement agricole lui donne le titre d'École nationale supérieure des industries agricoles et alimentaires (ENSIA), titre qu'elle va garder jusqu'en 2006.

Mais simultanément les autorités chargées de l'aménagement du territoire lui donnent à l'École, après plusieurs années d'incertitudes sur son installation en région parisienne une implantation définitive à Massy (Essonne) en prescrivant, toutefois, que n'y soit implantée qu'une seule année d'études. Cette restriction est interprétée par la communauté ENSIA comme un coup d'arrêt donné à son expansion.

\section{La mise en place de l'enseignement du génie des} procédés alimentaires en France (1961 - 2007)

L'implantation définitive de I'ENSIA en région parisienne permet aux responsables de l'école, délivrés de ce qui avait été leur souci_essentiel pendant la période précédente, de se consacrer à la mise en place de ce nouvel enseignement.

\section{L'enseignement de Marcel Loncin à I'ENSIA}

Ainsi que cela a été présenté ci-dessus, c'est le traité de 1961 qui a fait connaître Loncin. Toutefois il convient de rappeler que le directeur de I'ENIA, qui avait adopté dès 1952 la démarche du génie industriel et qui était donc intéressé par la notion d'u opération unitaire ", prend contact avec Loncini dés les années cinquante. La collaboration de ce dernier à l'enseignement 
de I'ENSIA se concrétise durant l'année scolaire 1962-1963 où il est définitivement chargé de ce cours. En venant dispenser son enseignement en France à I'ENSIA et non pas dans une autre institution, Loncin $a$, en quelque sorte, consacré cette école comme étant, en France, le lieu privilégié de cette nouvelle approche qui permet d'aborder scientifiquement toutes les techniques de transformation des produits agricoles en produits alimentaires. Marcel Loncin enseigne non seulement à Bruxelles mais également à I'Université de Karlsruhe, de 1972 à 1985. Tout ceci contribue à donner un rayonnement international à cette discipline.

Le génie des procédés alimentaires se développe rapidement à I'ENSIA, en particulier grâce à JeanJacques Bimbenet, originaire de I'ENSIA, recruté en 1962 et qui va traduire dans le quotidien de l'École les conceptions de Loncin. Bimbenet sera en 1995 le maître d'œuvre d'une troisième édition du traité de $1961^{13}$. Suivant l'évolution de la terminologie du génie chimique, le génie industriel alimentaire devient le génie des procédés alimentaires. On peut dater ce changement du premier congrès du génie des procédés, tenu à Nancy en 1986.

\section{L'institutionnalisation de l'approche transverse} Le congrès de I'International Union of Food science and Technology (IUFST), tenu en 1966 à Varsovie, recommande l'utilisation de l'approche transverse, c'est à dire par procédés et non par filières, pour l'enseignement des industries agroalimentaires. À l'ENSIA, c'est Jean Michel Clément (1934-1993), directeur de 1977 à 1987, qui en 1979 introduit cette approche en regroupant les enseignements en trois départements. Le premier est le génie industriel alimentaire devenu ensuite génie des procédés alimentaires, qui regroupe également les mathématiques, l'informatique et la mécanique. Le deuxième, la science des aliments est centrée sur la biochimie. Le troisième, la biotechnologie, comprend également la microbiologie industrielle. En 1986, est ajouté un quatrième département consacré à l'économie alimentaire. Ultérieurement, le département de biotechnologie est fusionné avec celui de science des aliments pour constituer ainsi, en parallèle à celui de génie des procédés alimentaires un enseignement tout à fait original.

13 Jean-Jacques Bimbenet et Marcel Loncin, Bases du génie des procédés alimentaires, Paris, Masson, 1995.
La signification de l'enseignement du génie des procédés alimentaires

On ne saurait trop souligner à quel point cette nouvelle discipline constitue l'aboutissement de l'évolution pédagogique de l'ENSIA et plus particulièrement du projet fondateur inscrit dans les termes même d'École des industries agricoles. Nous avons vu qu'à l'origine l'École avait été créée pour répondre aux besoins de la sucrerie et de la distillerie. Les circonstances de l'implantation de l'École dans le département du Nord avaient conduit à y ajouter l'enseignement de la brasserie-malterie. Ultérieurement étaient venues s'y ajouter d'autres filières de transformation de produit agricole en produit alimentaire telle que la cidrerie puis la laiterie. Le contexte des années qui ont suivi immédiatement la Deuxième Guerre mondiale, lequel a été exposé ci-dessus a conduit l'École à élargir encore son enseignement à des filières de transformation de produits déjà élaborés en produits alimentaires telles que la confiserie. ॥ est nécessaire de préciser que si certaines de ces filières avaient fait l'objet d'un approfondissement scientifique, telles que la sucrerie et la distillerie par l'Association des chimistes ou la brasserie et la distillerie par l'Institut Pasteur, beaucoup étaient fondées sur des savoirs demeurés empiriques. Or dés l'origine alors que l'enseignement ne portait que sur quatre filières, des redites étaient déjà apparues, certaines opérations se retrouvant dans plusieurs filières. Ces répétitions n'ont évidemment pu que se multiplier avec l'augmentation du nombre de filières enseignées. C'est pourquoi il est devenu nécessaire, dans les années qui ont suivi la Deuxième Guerre mondiale, d'adopter une autre démarche et d'isoler les opérations élémentaires constituant chacune des filières, telles que la filtration, l'évaporation, le séchage ou la distillation et d'approfondir chacune de ces opérations II était clair que le concept scientifique d'opération unitaire s'adaptait à cette situation. C'est pourquoi dans l'après-guerre et au début des années soixante, s'est progressivement élaboré dans l'enseignement un passage d'une approche par filières, correspondant chacune à un métier, vers une approche par procédés ou techniques transverses. Le génie des procédés alimentaires a permis, ainsi une approche scientifique des industries agricoles et alimentaires alors qu'auparavant beaucoup de ces filières étaient constituées de techniques d'origine empirique. En transposant les concepts d'origine nord-américaine au contexte européen, Marcel Loncin a théorisé ce nouvel enseignement et a apporté ainsi un renversement essentiel en élaborant ce qu'on peut appeler une véritable 
syntaxe des industries alimentaires. On ne saurait trop souligner l'importance de l'apport conceptuel que représente le génie des procédés alimentaires qu'en rappelant, ainsi qu'il a été exposé ci-dessus, qu'une tentative d'approche scientifique avait eu lieu à l'initiative d'une très grande institution scientifique, en l'occurrence l'Institut Pasteur mais qu'elle n'avait pas été plus loin qu'un rapprochement entre la brasserie et la distillerie; Au sein de l'ENSIA, l'approfondissement du génie des procédés alimentaires se poursuit ainsi qu'en témoignent des publications récentes ${ }^{14}$.

Cette nouvelle discipline a eu également un prolongement institutionnel constitué par la création en 1981 de l'Institut supérieur de l'agroalimentaire (ISAA). qui a établi une coordination dans l'enseignement du génie des procédés alimentaires et plus généralement dans celui d'une science pour l'industrie alimentaire, de deux autres écoles outre I'ENSIA, à savoir: I'Institut national agronomique ParisGrignon (INA-PG) et l'École nationale du Génie rural, des eaux et des forêts (ENGREF) Cette discipline a pris une nouvelle ampleur grâce à l'œuvre scientifique de l'économiste Louis Malassis qui a substitué au concept d'économie rurale, dominant jusque là, celui d'économie agro-alimentaire. On peut également faire l'hypothèse que la crise économique révélée par le premier choc pétrolier a incité les pouvoirs publics à prendre davantage conscience de l'importance de l'enseignement de ces techniques. Ce sont ces préoccupations qui vont se traduire, par la création de cette institution, dans laquelle l'ENSIA joue un rôle essentiel.

Mais le prolongement institutionnel le plus important est la fusion, en 2007, de I'ENSIA avec I'INA-PG et L'ENGREF cités ci-dessus, pour former I'Institut des sciences et industries du vivant et de l'environnement dénommé AgroParisTech. Ce nouvel établissement qui est donc le prolongement de I'ISAA, apporte ainsi à cet enseignement un cadre institutionnel beaucoup plus large.

\section{Conclusion}

Nous venons de voir que la transposition du génie chimique et plus particulièrement de la notion d'opérations unitaires à permis une approche scientifique des techniques utilisées dans l'industrie alimentaire. Or il s'agit d'une discipline scientifique élaborée tout à fait indépendamment de ce champ d'activité. L'histoire de l'institution dans laquelle

14 Jean-Jacques Bimbenet, Albert Duquenoy, Gilles Trystram, Génie des procédés alimentaires. Des bases aux applications. Paris, Dunod, 2002, 2e édition 2007. a émergé cet enseignement et la nature de son objet nous suggèrent plusieurs pistes de recherche, principalement issues des sciences humaines, pour l'approfondissement de l'enseignement de cette industrie. Plusieurs épisodes de l'histoire de I'ENSIA manifestent incontestablement un statut dévalorisé du savoir relatif à l'alimentation. Que ce soient les promoteurs de l'École, à l'origine, ou par la suite, les responsables de l'École dans des conditions parfois difficiles, ont souhaité une implantation en région parisienne. Les exemples du scepticisme rencontré par les responsables de l'École de la part des plus hautes instances scientifiques sont nombreux. Nous n'en citerons que deux.

En 1956, l'ENSIA ayant obtenu la construction d'un pavillon à la Cité universitaire de Paris, le directeur, venu présenter le programme et les buts de l'École devant la conférence des directeurs de fondation, s'est heurté au sourire ironique de ses collègues qui ne comprenaient pas que l'on puisse enseigner de la physique, de la chimie et de la biologie pour fabriquer des biscuits, de la confiture et du saucisson. En 1961, c'est l'éditeur qui a imposé à Marcel Loncin le titre de son ouvrage en estimant qu'une référence au domaine alimentaire serait peu attractive alors que la texte ne porte que sur l'industrie alimentaire. Cependant, en 1976, cet ouvrage sera réédité sous le titre de Génie industriel alimentaire. Cependant, il convient de signaler qu'un rapport, établi en 1984 sur l'enseignement agricole, donnait une appréciation plus flatteuse de l'enseignement alimentaire.

Nous proposons comme explication à cette situation l'hypothèse que l'alimentation était traditionnellement l'apanage des femmes qui, jusqu'à une date récente, avaient un statut dévalorisé dans la société française. Cette situation pourrait être approfondie, en se référant, en particulier, à la problématique élaborée par le philosophe allemand Axel Honneth. ${ }^{15}$ Par ailleurs l'ouvrage précurseur de Lindet pourrait être réédité. En effet, le manque de reconnaissance de cet ouvrage lors de sa publication est un autre aspect de ce statut dévalorisé.

En 2010, il ne paraît pas inutile de revenir sur le lien qu'on peut établir entre les crises économiques, d'une part, etl'histoire de l'ENSIA. et celle de l'industrie alimentaire, d'autre part.. Trois crises économiques majeures ont ponctué la vie de l'École : la longue

1516 Axel Honneth, La lutte pour la reconnaissance, Paris, Les éditions du Cerf, 2008. Nous nous devons de signaler que cette problématique a été présentée par André Grelon, directeur d'études à l'EHESS au cours de son séminaire consacré à la Sociologie des professions techniques durant l'année 2008-2009. Voir également la revue Esprit, juillet 2008. 
stagnation (1873-1897), la grande dépression des années trente et enfin le choc pétrolier.des années soixante-dix. Nous donnerons successivement cidessous, pour chacune de ces trois crises, d'une part, l'événement qui a fait prendre conscience publiquement de son existence et, d'autre part, un événement majeur intervenu dans la vie de l'École. Pour la longue stagnation, il s'agit du krach de l'Union générale en 1882 alors que le premier communiqué ministériel annonçant la création de l'École de Douai date de 1887. Ce communiqué marque, en quelque sorte, le point de non-retour de la puissance publique en faveur de la création de cette école. Pour la Grande dépression, le 24 octobre 1929, resté dans I'histoire sous le nom de Jeudi noir marque la prise de conscience aux Ełats-Unis de la crise alors qu'en 1934 Etienne Dauthy est nommé à la direction de l'École. Il convient de préciser à ce sujet que c'est Dauthy qui, en 1940, prendra la décision d'installer l'École à Paris et qui, en tant que directeur général, en suivra le développement jusqu'au début des années cinquante, donc pendant une période essentielle à son développement. Pour le Choc pétrolier dont la première date de 1973, rappelons que c'est en 1979 qu'est prise la décision de créer I'ISAA, déjà évoquée, lequel renforce le rôle de I'ENSIA. Le fait que les décisions de 1979 aient été appliquées après l'alternance politique de 1981 souligne qu'il ne s'agissait pas d'un enjeu idéologique, mais que c'était effectivement la crise qui en était la cause profonde. On constate qu'à ces trois reprises, le nombre d'années écoulées entre ces événements est similaire : cinq ans dans les deux premiers cas et six dans le dernier. On peut proposer comme explication que la collectivité, quand elle se sent atteinte, éprouve le besoin de se recentrer sur une activité considérée comme fondamentale mais négligée en période de prospérité. On peut ainsi noter le fait que le génie des procédés alimentaires, ou du moins la discipline et l'institution qui lui ont donné naissance, se sont particulièrement développés pendant les deux dernières guerres mondiales. 